\title{
Monte Carlo Studies of Quantum Cascade Lasers
}

\author{
P. BorowiK ${ }^{a}$, J.L. Thobel ${ }^{b}$, M. BugajSki $^{a, c}$ AND L. AdAmowicz ${ }^{a, *}$ \\ ${ }^{a}$ Wydział Fizyki Politechnika Warszawska, Koszykowa 75, 00-662 Warsaw, Poland \\ ${ }^{b}$ Institut d'Electronique et de Nanotechnologie, UMR CNRS 8520, Université Lille 1 \\ Avenue Poincaré, B.P. 69, 59652 Villeneuve d'Ascq Cedex, France \\ ${ }^{c}$ Instytut Technologii Elektronowej, al. Lotników 32/46, 02-668 Warsaw
}

\begin{abstract}
Results of multi particle Monte Carlo studies of a mid-infrared QCL structure, for the first time fabricated by Page et al., are presented. It has been demonstrated that for the considered structure design at least 9 electron energy levels per laser segment should be used to correctly describe the electric current properties. Mechanisms responsible for electron population on higher laser level are discussed.
\end{abstract}

PACS numbers: 72.10.-d, 72.20.Jv, 73.63.-b, 78.67.-n

\section{Introduction}

Over the past several years, quantum cascade lasers (QCLs) have proved to be one of the best avenues for developing compact, low consumption solid-state sources in the mid and far infrared spectral region. QCLs are unipolar semiconductor lasers based on transitions between sub-bands in a multi-quantum-well structure. Therefore, the lasing frequency can be varied in a wide range through a careful design of epitaxial structure. However, the complexity of QCLs makes highly desirable simulation tools that can deal with the complicated physical phenomena involved. One of the most powerful tools for investigating charge transport in semiconductors is the Monte Carlo method [1]. Adapting Monte Carlo simulation to QCL is not a trivial task. First of all, since electrons move in the direction perpendicular to heterojunctions, one needs to face a quantum transport problem. However, it has been shown that a Boltzmann-like formalism, neglecting coherent carrier dynamics, is sufficient at least for the stationary state [2]. With such an approach, the major problem arises from the huge number of possible scattering paths. This question is especially severe for terahertz QCL due to the large number of states involved in the transport process.

In this paper we present our results of Monte Carlo studies of the structure firstly proposed by Page et al. [3] and recently fabricated at IET, Warsaw [4]. Electron energy levels and wave functions are obtained by self-consistently solving Schrödinger and Poisson equations. However the doping level and electron density assumed for this simulation are small, therefore the space charge effect is negligible. During the simulation we used 15 en-

* corresponding author; e-mail: adamo@if.pw.edu.pl ergy levels per laser segment, so when 3 consecutive segments were simulated, in total 45 levels have been used. The model includes scattering mechanisms on acoustic and polar optical phonons as well as alloy scattering.

\section{Model and results}

The crudest approximation in our model is that it does not account for electron-electron scattering. This interaction may be the major mechanism governing the coupling between closely spaced sub-bands and it is thus known to play a major role for QCL operating at $\mathrm{THz}$ frequency. Sophisticated models have been developed to take it into account [5], unfortunately, they are very computer intensive. For the QCL considered here, which operates in mid-infrared range, we have neglected electronelectron scattering and we will discuss the consequence of this approximation.

In Fig. 1 we present the shape of structure potential and the calculated wavefunctions. Laser action should occur between 4 and 2 levels. The figure indicates that the levels above 9th not only have much higher energies in comparison to lower levels, but they are also more similar to continuum-like than to bound states. The energy separation prohibits scatterings to these levels within the same laser segment. Moreover, the scatterings to these states in the neighboring segment are not frequent because wave functions only weakly overlap, which is predominant factor in scattering probability.

One of the main outputs of Monte Carlo simulations of QCL structures is sub-bands population, presented in Fig. 2. For both studied temperatures our results show that significant electron occupation can be expected only for first five levels. Our other results demonstrate that the role of 6 to 9 levels should not be neglected. Electrons 


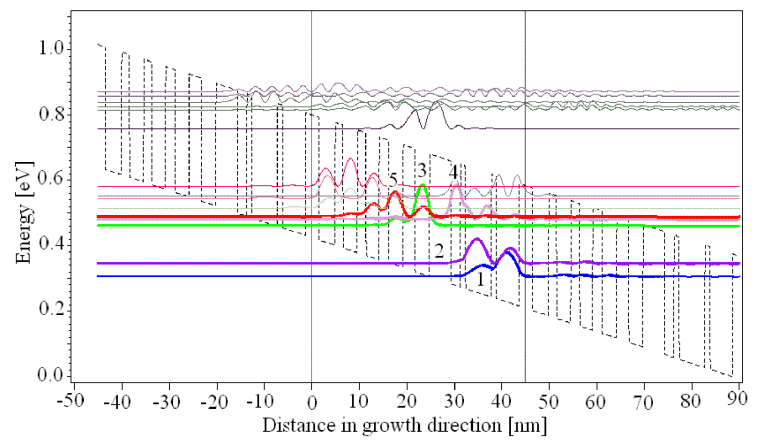

Fig. 1. Calculated square moduli of wave-functions for central segment, shifted to show the position of respective energy level. Labels (1-5) indicate the first 5 wave functions belonging to the central segment. The bottom of the conduction band, representing the studied structure potential, is drawn for three consecutive laser segments. The layer thickness sequence is as follows: $2.8 / \mathbf{3 . 4} / 1.7 / \mathbf{3 . 0} / 1.8 / \mathbf{2 . 8} / 2.0 / \mathbf{3 . 0} / 2.6 / \mathbf{3 . 0} /$ 4.6/1.9/1.1/5.4/1.1/4.8 [nm]. The thicknesses for GaAs layers are highlighted by bold type. Other layers are fabricated of $\mathrm{Al}_{0.45} \mathrm{Ga}_{0.55}$ As. Applied electric field intensity is equal to $48 \mathrm{kV} / \mathrm{cm}$.

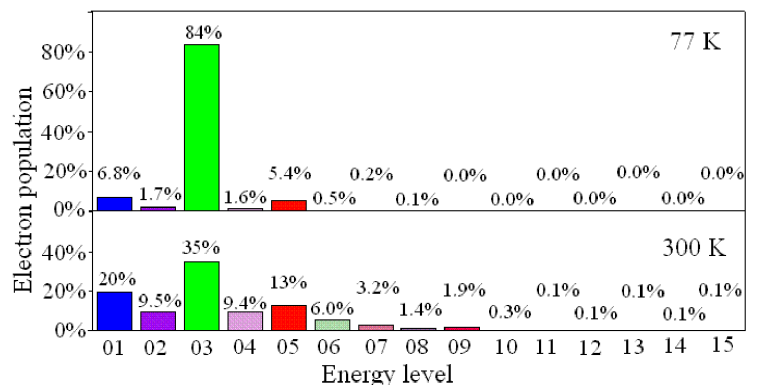

Fig. 2. Calculated levels population for $77 \mathrm{~K}$ and $300 \mathrm{~K}$. Significant electron occupation is visible for five lowest energy levels only. However, levels from 6th to 9th show their importance for higher temperatures. Accumulation of electrons on the 3rd level is explained in the text.

are scattered there and the current flowing through this channel is important.

Unfortunately, our model is not able to correctly describe all simulated structure characteristics. For example, we do not observe population inversion between lasing levels. We believe that this behaviour can be attributed to the lack in our model of Coulomb interactions leading to electron-electron $[3,6]$ and electron-impurity [7] scattering. Energy separation between 3rd and 4th sub-band $(20 \mathrm{meV})$ as well as between 4 th and 5 th sub-band $(8 \mathrm{meV})$ is quite small and there is a finite probability for electrons to be quasi-elastically scattered between these levels.

An interesting effect of accumulation of carriers on the 3rd level can be observed (Fig. 2). This may be explained when we notice that energy separation between this level and the next lower level (8th level from the right neighboring segment, not shown in the diagram from Fig. 1) is approximately $100 \mathrm{meV}$. This exceeds much phonon energy and probability of scattering to that level is relatively small. Although the electrons present at the 3rd level have much higher energies at the arrival, they are still trapped after thermalizing by optical phonons emision. For higher tempearture, when thermal energy $(k T=26 \mathrm{meV}$ for $300 \mathrm{~K})$ is close to levels' separation, there is much higher chance for electrons to be scattered out.

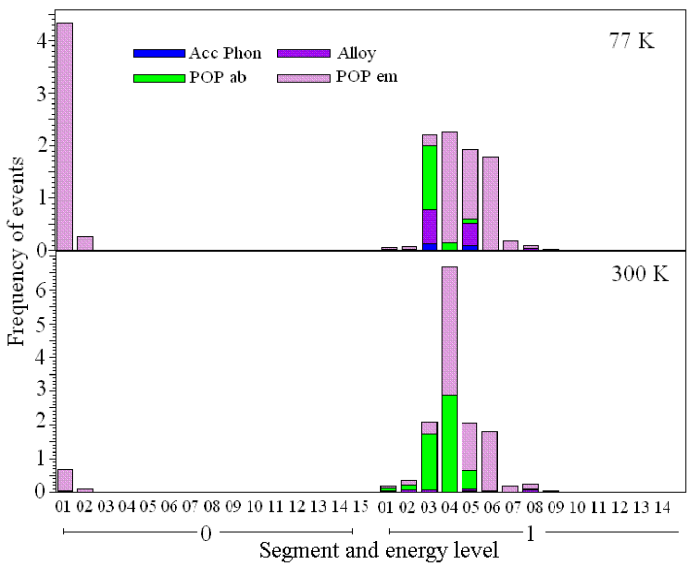

Fig. 3. Relative frequency of electron scatterings from all energy levels responsible for occupation of the single 4th level (higher lasing level) at $77 \mathrm{~K}$ and $300 \mathrm{~K}$. Only central and left laser segments are presented as there are no scatterings from the right segment to this state. The left group denoted by 0 represents levels attributed to left side neighboring segment of the studied laser segment. The right group denoted by 1 represents levels of the laser segment which is studied. Polar optical phonon (POP) absorption and emission, acoustic phonon and alloy scattering mechanisms are included.

One of the aims of the considered structure design is to obtain significant population inversion between levels 4 th and 2nd. As we already noticed, our model is not sufficient to demonstrate this behavior. Nevertheless it may be interesting to see which levels and what types of interactions contribute to filling upper lasing level. In Fig. 3 we present the starting levels for electrons that are scattered to the 4 th level. It is interesting to notice that for $77 \mathrm{~K}$ majority of electrons that occupy this level were scattered from the 1st level of the left neighboring segment. This level was designed to collect electrons from lower lasing level (2nd). The situation is different at $300 \mathrm{~K}$, when majority of electrons present in 4 th level come from the closests levels - 3rd, 5th and 6th. For both temperatures one can notice quite significant bar representing intra-sub-band scatterings when both initial and final state correspond to the 4 th level. For $77 \mathrm{~K}$ we observe mainly emissions of polar optical phonons, but for $300 \mathrm{~K}$ proportion of emissions and absorptions is almost equal which signifies that the quasi-equilibrium 
distribution is achieved. Obviously in the case of laser operation there will also be present a mechanism of radiative interaction leading to much shorter electron lifetime on this level.

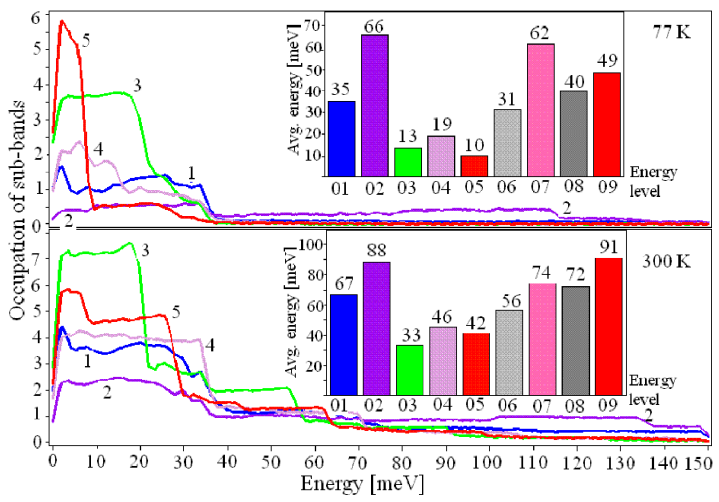

Fig. 4. Electron distribution functions $f(E)$ for the first five energy sub-bands (labeled from 1 to 5 ) for 77 $\mathrm{K}$ and $300 \mathrm{~K}$. Average electron energies for the first 9 sub-bands are presented in the inset.

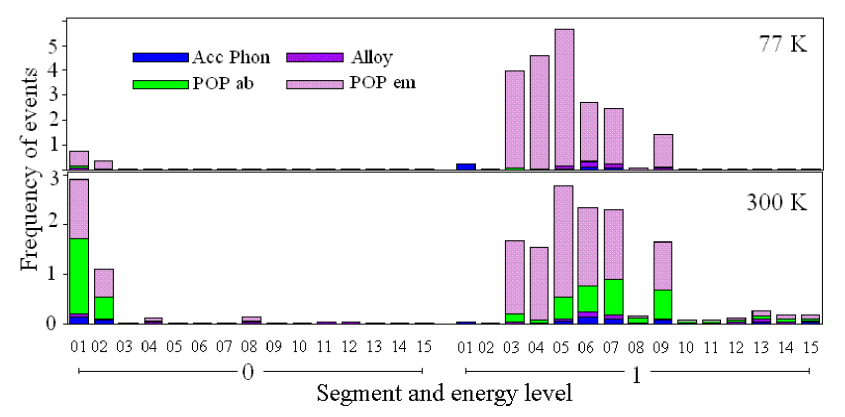

Fig. 5. Relative frequency of scatterings for which the final state is located in a neighboring segment. X-axis represents target level of the scattering. Polar optical phonon (POP) absorption and emission, acoustic phonon and alloy scattering mechanisms are included. The left group denoted by 0 represents scatterings to the left neighboring segment which is equivalent to backward current. The right group denoted by 2 represents scatterings to the right neighboring segment which is equivalent to forward current. Levels from 3rd to 9th participate in the forward charge transport for both temperatures.

Our modeling also confirms that the lower lasing level (2nd) is emptied by scattering to the 1 st level with emission of polar optical phonons, while other channels collecting electrons from this level are almost negligible. The same effect can be observed for both studied lattice temperatures. We do not present these findings on a separate figure as it would show almost only two big bars, one representing intra-sub-band scattering for electrons relaxing without changing the sub-band and the second bar for scatterings to the 1st level within the same segment.
It may be interesting to discuss the electron energies on consecutive levels of the structure. One can notice in Fig. 4 that we obtain perceptibly lower average energy on $3 \mathrm{rd}, 4 \mathrm{th}, 5$ th levels comparing to others. This can be due to the fact that electrons arrive at each level with significantly high energy, much higher than the thermal equilibrium. On these three particular levels they remain longer and have enough time to a relax to lower energy by emiting phonons. This behavior is also confirmed when we observe how energy distribution function looks like. For these three levels it tends to be more concentrated close to lower energies. On the contrary, when we look at the energy distribution for electrons occuping 2nd level we can see that it is more spread toward higher energy region.

Let us see which energy levels are in use by electric current flowing across the structure. In our Monte Carlo model the current is attributed only to scatterings to levels belonging to neighboring laser segment. Figure 5 presents target levels for such events. As one can notice, for both temperatures, the levels up to 9th are important. For higher temperature backward current also should not be neglected.

\section{Conclusions}

In the presented paper we demonstrated that Monte Carlo studies of QCL structures can give valuable insight into the description of electron transport behavior in the QCL structure. We were able to demonstrate which sub-bands should be included in the modeling. This was especially interesting to notice that even if the population on some levels seems to be negligible, the contribution to current flowing through them was remarkable. With the simplified physical model, neglecting Coulomb scattering, not all the important features could be predicted. For instance it was impossible to demonstrate population inversion between lasing levels.

\section{Acknowledgments}

The work was financially supported by grant PBZ-MNiSW-02/I/2007

\section{References}

[1] C. Jacoboni, L. Reggiani, Rev. Mod. Phys. 55, 645 (1983).

[2] R.C. Iotti, F. Rossi, Phys. Rev. Lett. 87, 146603 (2001).

[3] H. Page et al. J. Appl. Phys. 78, 3529 (2001).

[4] K. Kosiel et al. Photonics Lett. Poland 1, 16 (2009).

[5] O. Bonno et al. J. Appl. Phys. 97, 043702 (2005).

[6] P. Harrison, R.W. Kelsall, Solid State Electron. 42, 1449 (1998).

[7] H. Callebaut et al., J. Appl. Phys. 84, 645 (2004). 